\title{
A Comparison Study of the Surface Scattering Models and Numerical Model
}

\author{
H.T. Ewe ${ }^{1}$, Joel T. Johnson ${ }^{2}$ and K.S. Chen ${ }^{3}$ \\ ${ }^{1}$ Faculty of Engineering, Multimedia University, \\ Cyberjaya 63100, Selangor, MALAYSIA \\ Email: htewe@mmu.edu.my \\ ${ }^{2}$ Department of Electrical Engineering and Electro-Science Laboratory, \\ The Ohio State University, Columbus, OH 43210 USA \\ ${ }^{3}$ Center for Space and Remote Sensing Research and Institute of Space Science, \\ National Central University, Chung-Li, Taiwan 32054
}

\begin{abstract}
This paper describes a comparison study of surface scattering models and a numerical model for dielectric surfaces. Two surface scattering models namely the Integral Equation Model (IEM) and the Small Slope Approximation (SSA) model are used to calculate the backscattering coefficients of rough surfaces and the results are compared with numerical simulations based on the Moment Method (MoM). Analysis of the results obtained is also presented.
\end{abstract}

\section{INTRODUCTION}

In the study of the interaction of microwaves with media bounded by rough interfaces, a practical and realistic surface scattering model is required to understand the surface scattering mechanism and predict the scattering returns for a variety of surface profiles. The usability and correctness of the model are essential in microwave remote sensing and a good understanding of the validity and limitation of the models will be necessary for modeling active and passive microwave scattering mechanisms for earth terrain and also inversion models. Surface scattering models based on both the small perturbation method (SPM) and the Kirchhoff model had been widely used in the past in the theoretical modeling of microwave remote sensing [1]. Recent models such as the Integral Equation Model (IEM) [2] and the Small Slope Approximation (SSA) model [3] have shown great promise in the prediction of surface scattering returns from various research studies. In this paper, a comparison study of the IEM model and the SSA model is carried out for dielectric rough surfaces. To compare the predictions of backscattering coefficients from these two models with the actual backscattering returns from the surface, numerical simulations based on the Moment Method (MoM) are generated for the comparison. It is generally found that both the IEM and the SSA model with the inclusion of up to second order surface slope are good models in comparison with the predictions from the numerical models. The results of this study also provide useful information for the suitable applications of the two models in practical surface scattering calculations.

\section{CONFIGURATION}

Fig. 1 shows the configuration of the study where $\varepsilon_{r}$ is the complex dielectric constant of the surface and $\theta_{i}$ is the incident angle. A 2-D (3-D scattering problem) isotropic Gaussian correlated surface with surface rms height $\sigma$ and surface correlation length $l$ is considered.

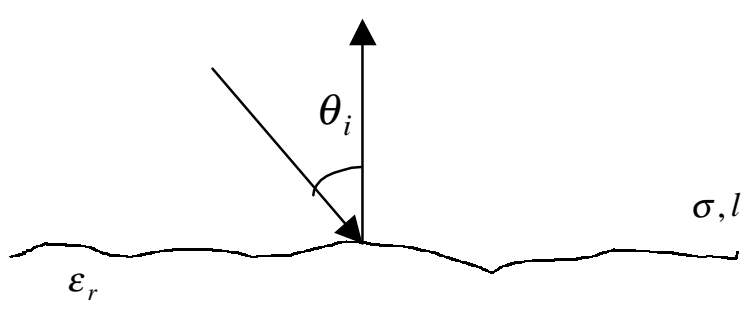

Fig 1. Surface configuration

\section{SURFACE SCATTERING MODELS}

\section{a. Small Slope Approximation (SSA) Model}

The Small Slope Approximation (SSA) [3] is chosen for the comparison as recent studies have shown that this is a robust model for surface scattering. The SSA is based on a series expansion in terms of surface "quasi-slope", with evaluation of average incoherent cross sections from the first term (first order in slope) requiring a calculation similar to that of the Kirchhoff approximation but retaining agreement with perturbation theory in the small height limit. Expressions for average cross sections accurate to second order in slope are also available [3] but require additional integrations. An alternate approach for evaluation of higher order corrections is described in [4] based on Monte Carlo simulation. The Monte Carlo procedure is applied in this paper, with averaged second order in slope cross sections generated from 100 realisations of 128 by 32 lambda periodic 
surfaces sampled into 1024 by 256 points under plane wave incidence. Because the higher order SSA calculation can be implemented with the Fourier transform, the computation is very efficient compared to a Monte Carlo simulation with the Method of Moments.

\section{b. Integral Equation Model (IEM)}

For the Integral Equation Model (IEM) in [2], it is known that the model provides a good prediction of surface scattering coefficients for a wide range of surface profiles which include the limits of both the classical Kirchhoff model (KM) and the small perturbation model (SPM). In the expression of the IEM model, three terms of surface scattering contributions, namely the Kirchhoff term $(k)$, the cross term $(k c)$ and the complementary term $(c)$ are included and shown in (1), respectively:

$$
\sigma_{q p}^{o}=\sigma_{q p}^{k}+\sigma_{q p}^{k c}+\sigma_{q p}^{c}
$$

where $\sigma_{q p}^{0}$ is the surface backscattering coefficient and the subscripts $q$ and $p$ denote the scattered polarisation and the incident polarisation, respectively. In this study, an improved model of IEM is used where this model is included with the empirical reflection coefficient model [5] that provides smooth transition in the Fresnel reflection coefficient approximation across a range of angular and frequency conditions.

\section{c. Method of Moments (MOM)}

Approximate model results are compared with an "exact" numerical model based on Monte Carlo simulation with an iterative method of moments based surface scattering computation. Computational time for the numerical model is reduced through the use of the "canonical grid" algorithm for computing matrix-vector multiplies to order $(\mathrm{N} \log \mathrm{N})$ where $\mathrm{N}$ is the number of surface sampling points. A detailed description of the algorithm is provided in [6]-[7]. To further keep computational times reasonable, numerical results use 32 realisations of $16 \times 16$ lambda surfaces sampled into 128 by 128 points, and the Monte Carlo simulation is performed through the use of a IBM P2SC parallel computing resources at the Maui High Performance Computing Center [8]. Due to the finite size of the surfaces modeled in the MOM, a "tapered-wave" incident field is used to avoid artificial surface edge scattering effects. The tapered wave and surface size chosen make MOM results accurate only to up to incidence angle 50 degrees, so the comparison is not continued outside this range. Numerical model results are also not included for incidence angle 0 degrees due to the dominance of the coherent scattered field at this angle for the surface statistics considered.

\section{RESULTS AND DISCUSSION}

In this study, a Gaussian correlated surface with complex dielectric constant $4+\mathrm{j} 1$ is used. A surface profile with $k \sigma=$ $0.5, k l=3.0$ is chosen where $k$ is the wave number. Note these surface statistics have only a moderate roughness, but the relatively large slopes encountered make the SPM and Kirchhoff models inaccurate here. Backscattering coefficients are compared for the IEM and both the first and second order SSA. In the figures, only second order SSA results are shown together with the IEM and numerical calculations.

Fig. 2 show the comparison of the VV backscattering coefficients calculated from the IEM, SSA model (second order) and numerical model. It is found from the calculations that the SSA model (first order) predictions are correct only for low incident angle $\left(<20^{\circ}\right)$. For higher angles than this, its predictions are generally lower than those of the other models. However, the second order SSA, IEM, and numerical model all show good agreement for the range of angles considered. Although the data for numerical model is only up to $50^{\circ}$, it is found that both the IEM and SSA model continue to show agreement up to $70^{\circ}$.

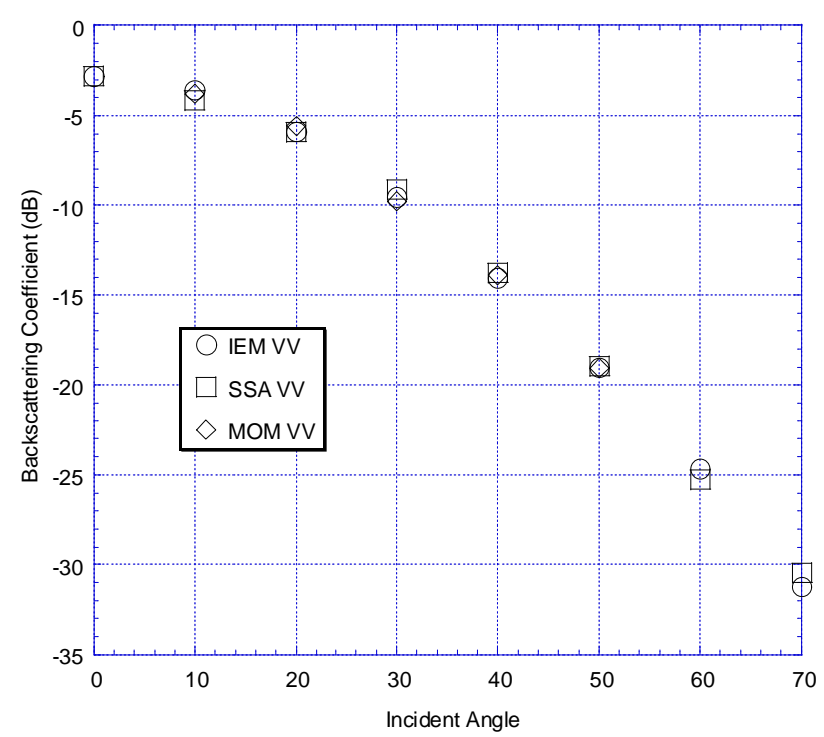

Fig 2. Comparison of the calculations of different surface models for VV polarisation $(k \sigma=0.5, k l=3.0)$. 
In Fig. 3, the same comparison is presented for $\mathrm{HH}$ polarisation. It is again found that the SSA model (first order) calculations are accurate only for low incident angles and generally give higher backscattering coefficients for high incident angles as compared with the other models. The match between the SSA model (second order) calculations and the numerical model calculations is again very good and those of the IEM also compare very well with the numerical models for the particular problem considered in this example.

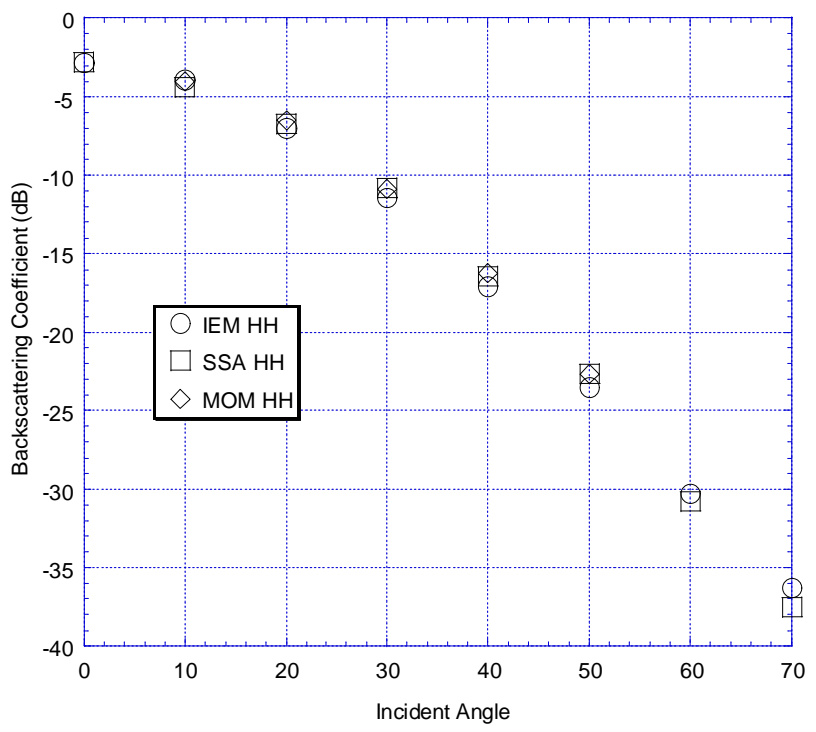

Fig 3. Comparison of the calculations of different surface models for $\mathrm{HH}$ polarisation ( $k \sigma=0.5, k l=3.0$ ).

\section{CONCLUDING REMARKS}

Generally, it is found in this example that both the SSA model with up to second order terms of surface slope and the IEM compare well with the numerical calculations for likepolarisation in the backscattering directions. Additional examples and studies of bistatic cross sections are currently in progress.

\section{REFERENCES}

[1] F. T. Ulaby, R. K. Moore and A. K. Fung, A. K., Microwave Remote Sensing: Active and Passive (Vol. II). Reading, Massachusetts: Addison-Wesley, 1982

[2] A.K. Fung, Microwave Scattering and Emission Models and Their Applications, Artech House, Norwood, MA, 1994.
[3] A. G. Voronovich, Wave Scattering from Rough Surfaces. Berlin: Springer-Verlag, 1994.

[4] S. T. McDaniel, "Acoustic and radar scattering from directional seas," Waves in Random Media, vol. 9, no. 4, pp. 537--549, 1999.

[5] T. D. Wu, K. S. Chen, A. K. Fung and M. K. Tsay, “A Transition Model for the Reflection Coefficient in Surface Scattering", Proc. Of IGARSS'1998, pp. 23752377.

[6] J. T. Johnson, R. T. Shin, J. A. Kong, L. Tsang, and K. Pak, "A numerical study of ocean polarimetric thermal emission," IEEE Trans. Geosc. Remote Sens. vol. 37, no. 1 (part I), pp. 8--20, 1999.

[7] J. T. Johnson, R. T. Shin, J. A. Kong, L. Tsang, and K. Pak, "A numerical study of the composite surface model for ocean scattering," IEEE Trans. Geosc. Remote Sens., vol. 36, no. 1, pp. 72-83, 1998.

[8] Maui High Performance Computing Center WWW site, at http://www.mhpcc.edu 